\title{
Local Identification of Voltage Instability from Load Tap Changer Response
}

Weckesser, Johannes Tilman Gabriel; Papangelis, Lampros; Vournas, Costas D. ; Van Cutsem, Thierry

Published in:

Sustainable Energy, Grids and Networks

Link to article, DOI:

10.1016/j.segan.2017.01.001

Publication date:

2017

Document Version

Peer reviewed version

Link back to DTU Orbit

Citation (APA):

Weckesser, J. T. G., Papangelis, L., Vournas, C. D., \& Van Cutsem, T. (2017). Local Identification of Voltage Instability from Load Tap Changer Response. Sustainable Energy, Grids and Networks, 9, 95-103.

https://doi.org/10.1016/j.segan.2017.01.001

\section{General rights}

Copyright and moral rights for the publications made accessible in the public portal are retained by the authors and/or other copyright owners and it is a condition of accessing publications that users recognise and abide by the legal requirements associated with these rights.

- Users may download and print one copy of any publication from the public portal for the purpose of private study or research.

- You may not further distribute the material or use it for any profit-making activity or commercial gain

- You may freely distribute the URL identifying the publication in the public portal 


\title{
Local Identification of Voltage Instability from Load Tap Changer Response
}

\author{
Tilman Weckesser ${ }^{\mathrm{a}, *}$, Lampros Papangelis ${ }^{\mathrm{a}}$, Costas D. Vournas ${ }^{\mathrm{b}}$, \\ Thierry Van Cutsem ${ }^{\mathrm{c}}$ \\ ${ }^{a}$ Dept. Electrical Engineering $\&$ Computer Science at the University of Liège, Belgium \\ ${ }^{b}$ National Technical University of Athens, Athens, Greece \\ ${ }^{c}$ Fund for Scientific Research (FNRS) at the University of Liège, Belgium
}

\begin{abstract}
This paper presents a local long-term voltage instability monitoring method, which is suitable for on-line applications. The proposed extended-time Local Identification of Voltage Emergency Situations (eLIVES) method is a significantly modified version of the previously presented LIVES method. The new method is not bound to assessing system response over a predefined LTC tapping period. This allows handling LTCs with variable delays, as well as events taking place during the tapping sequence impacting the distribution voltages. For that purpose, eLIVES applies recursive least square fitting to acquired distribution voltage measurements and a new set of rules to detect a voltage emergency situation. The effectiveness of the eLIVES method is presented on the IEEE Nordic test system for voltage stability and security assessment.

Keywords: power system dynamics, voltage stability, on-line stability monitoring
\end{abstract}

\section{Introduction}

Since the blackouts that took place in the 80's (e.g. [1]), voltage instability in power systems has been intensively studied $[2,3$. Liberalization of the electricity

\footnotetext{
* Corresponding author

Email addresses: j.weckesser@ulg.ac.be (Tilman Weckesser),

1.papangelis@ulg.ac.be (Lampros Papangelis), vournas@power.ece.ntua.gr

(Costas D. Vournas), t.vancutsem@ulg.ac.be (Thierry Van Cutsem)

Preprint submitted to Sustainable Energy, Grids and Networks

January 6, 2017
} 
markets has put pressure on power systems and is likely to result in operating 5 conditions closer to stability limits in the near future. For this reason, an early and reliable identification of developing instabilities or emergency conditions will become crucial to operate power systems securely. A widespread practice for voltage stability monitoring is to observe the voltage magnitudes at certain key transmission buses and issue an alarm, when they drop below pre-defined critical thresholds. For this approach, a major challenge is to define appropriate thresholds valid for a wide range of disturbances. Recent research efforts have been focusing on more accurate on-line voltage stability monitoring and early detection of developing voltage instability. The aim of these methods as well as the one proposed in this paper is to identify an evolving instability before 15 it is apparent from sole observation of bus voltage magnitudes. A review of existing voltage instability detection methods has recently been published in [4. The developed methods are either attempting to detect voltage instability on a system-wide level or purely locally. Detection methods utilizing widearea measurements are, for example, based on decision trees [5] or suitable voltage stability indices [6, 7, 8, Some of the system-wide methods require full system observability obtained from PMUs, which is not yet available. However, a combination of PMUs with SCADA measurements can already today provide full system observability.

Local methods require only locally available measurements, which are easily accessible. A group of local methods attempts to determine voltage stability through detection of the impedance matching condition [9, 10, 11]. Another local method, called Local Identification of Voltage Emergency Situations (LIVES) [12, 13, is utilizing measurements, which are readily available in the Load Tap Changer (LTC) controlling bulk power delivery transformers. In order to early detect voltage instability, LIVES solely monitors the voltage magnitude on the controlled, distribution side of the transformer. This voltage is not only affected by the local LTC, but reflects the combined effect of all voltage controllers in the system, e.g. other LTCs, OverExcitation Limiters (OELs) and shunt capacitor switching. Figure 1 shows an example of a voltage magnitude at a Medium 
35 Voltage (MV) bus, the ratio of the local transformer and the voltage magnitude at the corresponding High Voltage (HV) bus. It can be observed that the

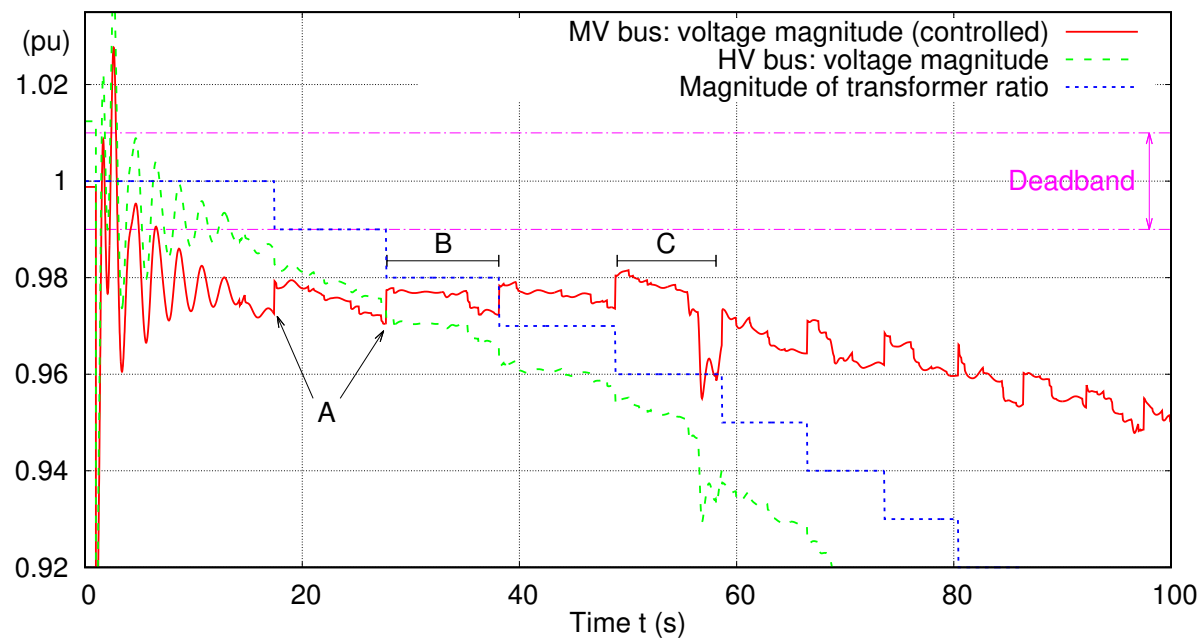

Figure 1: Example of combined effect of local LTC and other voltage controllers in a voltage long-term voltage unstable evolution after a line outage.

voltage magnitude at the $\mathrm{HV}$ bus is declining after the initial outage, under the effect of multiple tap changes (including the one shown in Fig. 1). After the initial oscillations of the MV voltage, induced by a fault and its clearance, 40 damped out, it can be observed that each tap change in the shown transformer (identified by the jumps in the dashed blue curve) temporarily increases the corresponding MV bus voltage magnitude (see $A$ in Fig. 1). This temporary increase is to some extent canceled by the effect of other tap changers as well as field current limiters. An almost complete cancellation of the benefit of the local tap change can be observed in the time interval marked $B$ in Fig. 1, while during the period marked $C$, the other tap-changes and field current limitations clearly prevail. From this time on, an overall negative trend of the distribution voltage magnitude can be observed, which is typical for long-term voltage instability.

The early detection of the above unsuccessful distribution voltage restoration ${ }_{50}$ is the topic of this paper. It should also be mentioned that, in near future, the response of active distribution grids will play a role in voltage recovery. However, 
in this paper the focus is on the effect of load tap changers.

The LIVES method described in [12] relies on the assumption that LTCs apply a constant tap delay. On the contrary, this paper proposes an approach that encompasses the case when LTCs have variable tap delays, e.g. obeying an inverse time characteristic, where the tap delay decreases with an increasing deviation of the controlled voltage from its set-point (as in the example of Fig. 11).

The development of the proposed extended-time LIVES (eLIVES) method was motivated by the idea of considering the whole distribution voltage recovery after an unforeseen event instead of looking at an incremental behavior over one tapping period.

The paper is structured as follows. In Section 2 the original LIVES method [12] is summarized and reasons for its non-applicability in the case of variabledelay LTCs are presented. In Section 3, the recursive least squares approach, which is used to fit a linear regression model to the voltage evolution at the LTC controlled bus, is explained. Then the rules used for the detection of a voltage emergency situation are introduced. The IEEE Nordic Test System used for the validation of the proposed method as well as the obtained simulation results of the identification of voltage emergency situations are discussed in Section 4 Section 6 presents an assessment of the methods robustness, when noise is added to the measurement samples. Finally, some concluding remarks are offered in Section 7 .

\section{Principle and limitations of the LIVES method}

The LIVES method [12] relies on the individual monitoring of distribution transformers, equipped with LTCs. Essentially, the method identifies an evolving long-term voltage instability through the fact that the positive effect on the controlled voltage of a local tap change is canceled by tap actions of other transformers and similar events in the system.

For that purpose, LIVES observes the controlled voltage over a time interval 


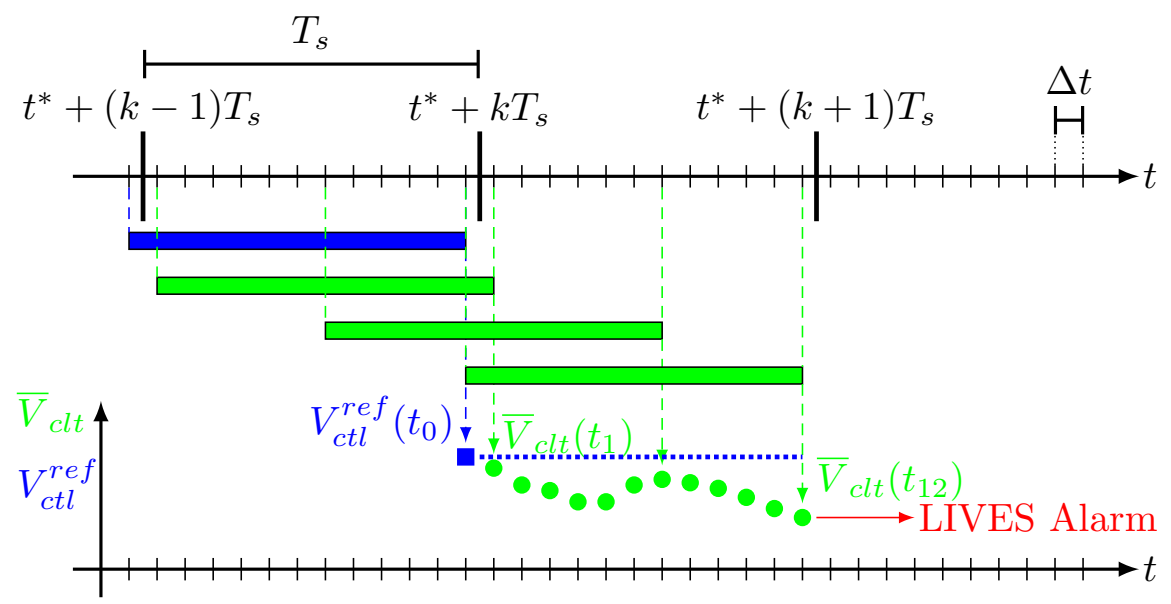

Figure 2: Visualization of the MA calculation in LIVES at an LTC with constant tap delay $T_{s}$

including a single tap change of the local transformer. To filter measurement noise and transients, a Moving Average (MA) of the voltage is computed. The MA's sliding window size is chosen equal to the time between two tap changes. Figure 2 is used to explain how MA computation and monitoring work. On the upper time line, the shorter vertical ticks indicate the discrete times, where voltage measurement samples are collected. The sample rate is $\Delta t$. The longer vertical ticks depict times at which tap changes occur in the monitored transformer. It is assumed that the LTC applies a constant delay $T_{s}$ between successive tap changes and that the controlled voltage does not re-enter the deadband during the displayed time period. $t^{*}$ corresponds to the time of the first tap change; hence, the subsequent tap changes occur at $t^{*}$ plus a multiple of $T_{s}$. The blue and green bars correspond to the time window, used to compute the respective MA. On the lower time line, the shorter vertical ticks again show the discrete times, where samples are collected. On the ordinate, the computed MA 95 of the controlled voltage $\left(\bar{V}_{c t l}\right.$, green circles) and a reference value $\left(V_{c t l}^{r e f}\right.$, blue squares) are shown.

The implementation of LIVES can be summarized as follows [13].

1. Before a tap change, e.g. at the discrete time $t_{0}$, the Moving Average 
$\bar{V}_{c l t}\left(t_{0}\right)$ of the controlled voltage $V_{c t l}$ is calculated as follows:

$$
\bar{V}_{c l t}\left(t_{j}\right)=\frac{1}{n} \sum_{k=0}^{n-1} V_{c t l}\left(t_{j}-k \Delta t\right)
$$

where $n$ is the number of samples involved. In order to ensure that only one tap change is included in the computation of the MA, $n$ is computed to correspond to the constant tap delay $T_{s}$ of the respective LTC:

$$
n=\left\lceil\frac{T_{s}}{\Delta t}\right\rceil
$$

This MA is stored as a reference value.

$$
V_{c t l}^{r e f}\left(t_{0}\right)=\bar{V}_{c l t}\left(t_{0}\right)
$$

2. At every sampling step $t_{j}=t_{0}+j \Delta t(j=1, \ldots, n)$ the MA is updated using (1). It is then compared to $V_{c t l}^{r e f}$. If the MA exceeds this reference, (indicating that voltage is recovering) no further check is going to be performed until a new reference is computed. On the other hand, if the MA remains below the reference for a period of at least $T_{s}$ an alarm is issued.

3. Before the next tap change and if the MA at some point exceeded the reference, a new reference is taken equal to the MA just before the tap and the monitoring is continued as described above.

In the example shown in Fig. 2, the reference $V_{c t l}^{r e f}$ is computed at $t_{0}$ and the subsequent MA $\bar{V}_{c t l}\left(t_{j}\right)$, which are computed with the samples in the respective window, are compared to it at times $t_{1}, \ldots, t_{12}$. In the shown case the MAs remain below the reference for the entire period $T_{s}$ and, hence, LIVES raises an alarm after $\bar{V}_{c t l}\left(t_{12}\right)$ has been evaluated.

It is apparent that for computing the MA and the reference value, the time until the next tap change needs to be known as well as the tap delay $T_{s}$. This information is easily obtainable, if the LTC applies a constant delay. However, if the delay varies (with the voltage deviation) the above MA comparison is no longer valid. Figure 3 shows an example, where the time $T_{2}$ between the second and third tap change is shorter than $T_{1}$ between the first and second tap change. 


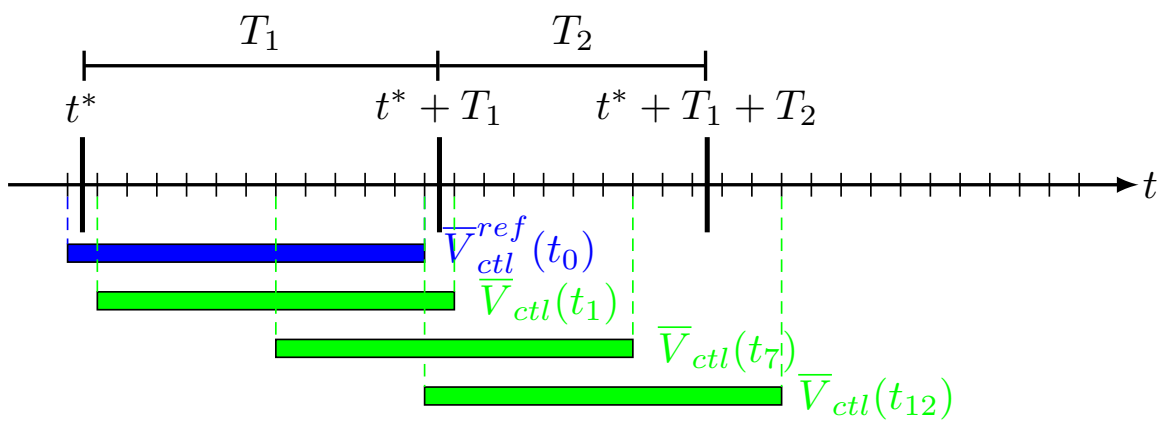

Figure 3: Visualization of the MA calculation in LIVES at an LTC with variable tap delay

If the MA window is chosen equal to $T_{1}$, then during the observation period some MAs, e.g. $\bar{V}_{c t l}\left(t_{12}\right)$ (see Fig. 3), will be computed based on samples containing two tap changes, which is overoptimistic. Alternatively, when the shorter tap delay is discovered, the MA window could be reduced to $T_{2}$ and the latest reference could be updated. However, to this purpose the new reference should be based on the period $T_{2}$. Since $T_{1}>T_{2}$, the samples used for this computation would not include a tap change and, hence, the assumption underlying the LIVES method would be violated again (this time being overly pessimistic).

To summarize, the underlying stability criterion of LIVES requires a synchronization with the LTC and the MA is a numerical filter appropriate only for fixed tap delays. These prerequisites are not fulfilled in the presence of LTCs with variable tap delay and, consequently, the LIVES method cannot be directly applied.

This limitation prompted the development of the eLIVES method described in the next section.

\section{Extended-time Local Identification of Voltage Emergency Situa- tions}

The eLIVES method is based on the detection of a decreasing trend of the secondary (controlled) voltage and a drop of the primary voltage below a predefined threshold during the post-disturbance evolution. The criterion uses 
only local available measurements and information, namely the primary voltage magnitude $V_{H V}$, the secondary voltage magnitude $V_{c t l}$, the width of the halfdeadband $\delta$, transformer ratio and maximum tap delay $T_{\text {max }}$, which corresponds to the maximum delay when $V_{c t l}$ is just outside of the deadband of the respective LTC.

The method is executed when a significant disturbance in the system is detected and the secondary voltage leaves the deadband, while the LTC is still active, i.e. it has not yet reached its hard tap limits. After the detection of the first tap change, the method begins to record voltage measurements. These measurements are utilized to identify the trend of the voltage evolution using a linear regression model.

\subsection{Recursive least square fitting of voltage measurements}

In each time step $t_{i}$, the estimator determines a linear regression model, which is assumed to show the overall trend of the monitored voltage:

$$
V_{f}(t)=m_{i} t+b_{i}
$$

In the post-disturbance configuration, secondary voltage samples are gathered to determine a linear (in time) approximation of the voltage evolution. For that purpose, the following equations need to be solved in least squares sense:

$$
\boldsymbol{V}_{i}=\left[\begin{array}{c}
V_{1} \\
V_{2} \\
\vdots \\
V_{i}
\end{array}\right]=\underbrace{\left[\begin{array}{cc}
t_{1} & 1 \\
t_{2} & 1 \\
\vdots & \vdots \\
t_{i} & 1
\end{array}\right]}_{\boldsymbol{A}_{i}} \underbrace{\left[\begin{array}{c}
m_{i} \\
b_{i}
\end{array}\right]}_{\boldsymbol{\theta}_{i}}
$$

where $\boldsymbol{A}_{i}$ is the regression matrix and $\boldsymbol{\theta}_{i}$ is the corresponding parameter vector determined at $t_{i}$.

Since the method assesses long-term voltage stability, which may require several minutes of monitoring after a disturbance, using a conventional least squares approach may not be feasible. For that reason, the model is fitted using 
a recursive least squares (RLS) approach [14, which has the advantage that, although all recorded measurements are considered, no large history needs to be processed at each time. The model is updated solely by processing the newest measurement. When a new measurement is received, the parameter vector $\boldsymbol{\theta}_{i}$ is updated by the RLS estimator as follows:

$$
\boldsymbol{\theta}_{i}=\boldsymbol{\theta}_{i-1}+\boldsymbol{R}_{i}^{-1} \boldsymbol{a}_{i}\left(V_{i}-\boldsymbol{a}_{i}^{T} \boldsymbol{\theta}_{i-1}\right)
$$

where $\boldsymbol{a}_{i}$ is the regressor $\left[\begin{array}{ll}t_{i} & 1\end{array}\right]$ and $R_{i}$ the covariance matrix at time $t_{i}$. The inverse of the covariance matrix can be directly updated according to:

$$
\boldsymbol{R}_{i}^{-1}=\boldsymbol{R}_{i-1}^{-1}-\boldsymbol{R}_{i-1}^{-1} \boldsymbol{a}_{i}\left(1+\boldsymbol{a}_{i}^{T} \boldsymbol{R}_{i-1}^{-1} \boldsymbol{a}_{i}\right)^{-1} \boldsymbol{a}_{i}^{T} \boldsymbol{R}_{i-1}^{-1}
$$

Since (7) allows directly updating the inverse of the covariance matrix, the inversion is performed only at the initialization. To compute a first estimate of the parameters, at least two successive measurements of the voltage magnitude are acquired, and the covariance matrix $\boldsymbol{R}_{i}$ is computed as follows:

$$
\boldsymbol{R}_{i}=\boldsymbol{A}_{i}^{T} \boldsymbol{A}_{i}
$$

In this paper, all measurements are considered and, hence, no forgetting factor was introduced.

The slope of the estimate $m_{i}$ is then used in the process of identifying a voltage emergency situation. Figure 4 shows two examples of the recorded volt-
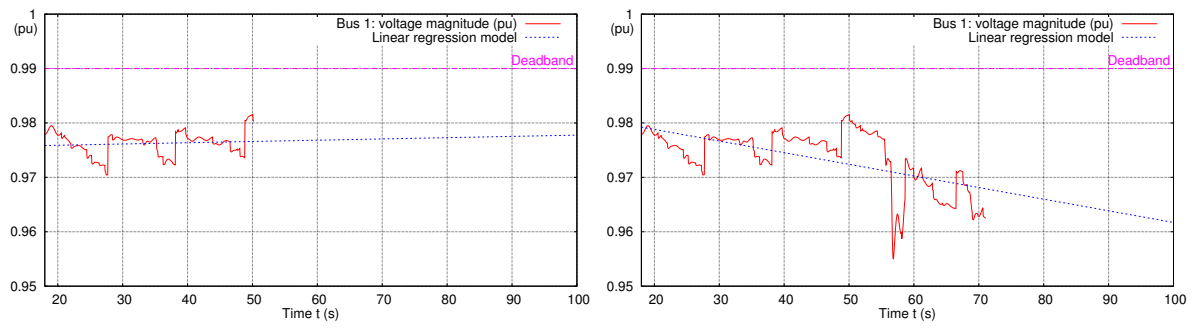
(a) At $t_{i}=50.2 \mathrm{~s}$ : Positive slope
(b) At $t_{i}=71.1 \mathrm{~s}:$ Negative slope $m_{i}=2.331 \cdot 10^{-5}$
$m_{i}=-2.137 \cdot 10^{-4}$

Figure 4: Comparison of the fitted linear regression model at two time instants. 
age and the respective fitted linear regression model. Since the aim is to assess the overall trend of the voltage, the RLS approach without forgetting factor is employed, which considers all measurements from the start of the monitoring (here $t=18.0 \mathrm{~s}$ ) and until the current time (in Fig. $4 \mathrm{a} 50.2 \mathrm{~s}$ and in Fig. $4 \mathrm{~b}$ $71.1 \mathrm{~s}$, respectively). In Fig. 4a a general improvement of the voltage magnitude and a positive slope of the fitted model can be observed, while Fig. 4b shows a declining voltage magnitude and a negative slope. Consequently, the proposed stability index is determined based on the monitored slope of the fitted linear regression model.

\subsection{Rules for identification of a voltage emergency situation}

In order to efficiently detect an emergency situation, a set of rules has been derived. The algorithm is activated when the controlled voltage leaves the deadband and voltage measurements are acquired to estimate the overall trend of the voltage. If a negative trend (declining voltage magnitude) is identified, an eLIVES Warning is issued. If additionally a drop of the voltage $V_{H V}$ on the primary side greater than a pre-defined threshold $\Delta V_{\text {crit }}$ is detected, an eLIVES Alarm is raised. The pseudo-code of the algorithm executed at time $t_{i}$ is shown next.

1: if $V_{c t l}\left(t_{i}\right)<V_{\text {set }}-\delta$ then

2: $\quad$ if recording $=$ off and tap change occurred then

175 $3: \quad$ recording $:=$ on; $t_{D B}:=0 ; t_{r e c}:=t_{i}$

4: $\quad$ else

5: $\quad$ if $t_{D B}>0.0$ then

6: $\quad t_{D B}:=0.0$

7: $\quad$ end if

180

8: end if

9: else

10: $\quad$ if recording $=$ on then

11: $\quad$ if $t_{D B}=0.0$ then 


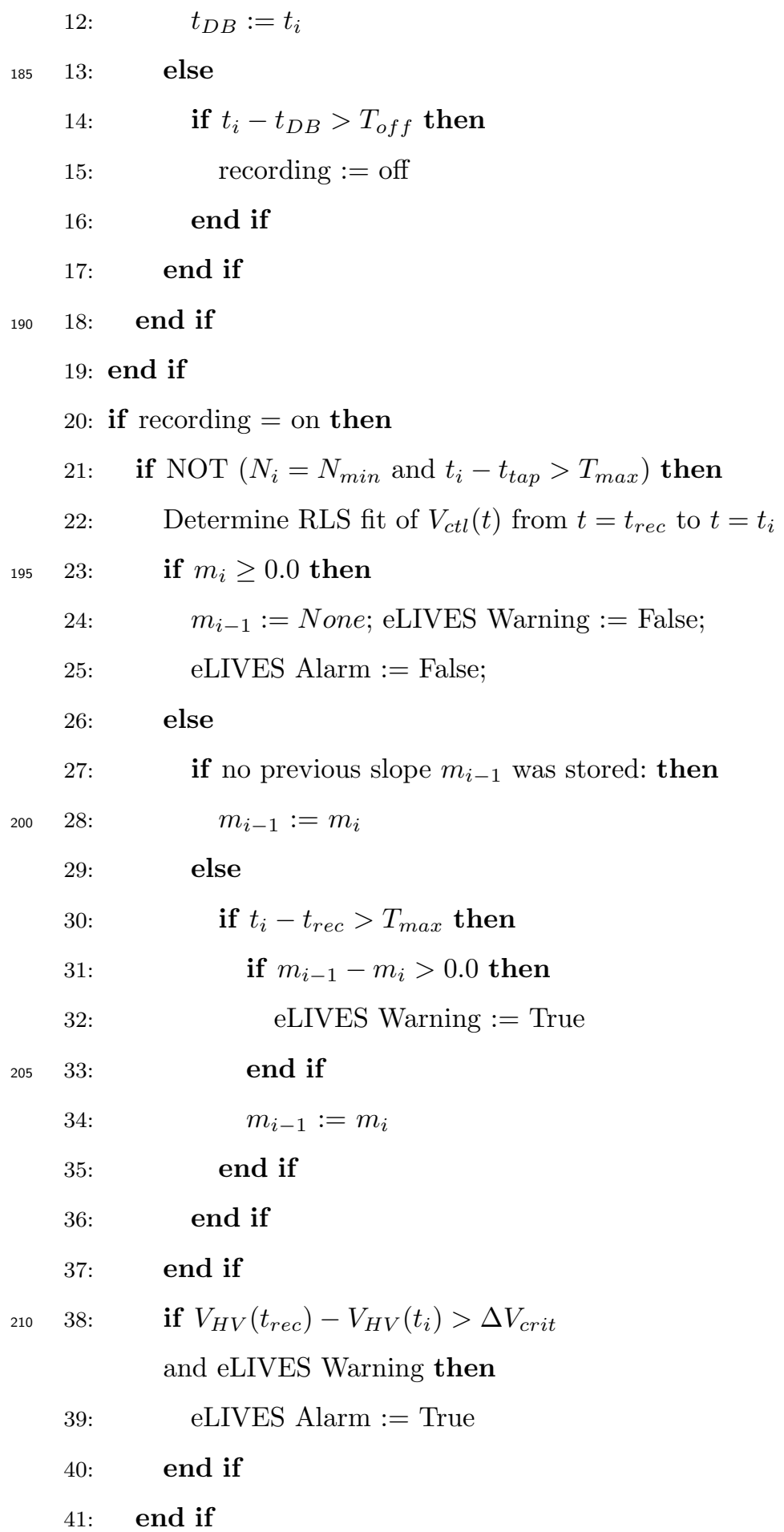


43: HV Alarm $:=$ True

44: end if

45: end if

The algorithm is stopped, when the voltage remains in the deadband for longer than $T_{o f f}$. In the following the algorithm will be explained in detail. In order to refer to parts of the algorithm, the text will refer to particular line numbers in the algorithm $(A L)$.

The beginning of the algorithm $(A L 1-19)$ shows the reasoning to determine if the monitoring with eLIVES should be started, continued or stopped. 225 If the voltage $V_{c t l}$ leaves the deadband $(A L 1)$ and a tap change was detected $(A L 2)$, the monitoring with eLIVES is activated at $t_{r e c}=t_{i}$. Then the method starts to record samples of the secondary voltage magnitude. Additionally, $t_{D B}$, which indicates the time when $V_{c t l}$ re-enters the deadband, is reset (see $A L 3$ ). The requirement of the detection of a tap change ensures that the monitoring is only activated, if the LTC is not yet at its limit. After activation, when the transformer ratio $N_{i}$ of the LTC reached its hard tap limit $N_{m i n}$, the monitoring with eLIVES is stopped after a delay equal to the respective maximum tap change delay $T_{\max }(A L 21)$. eLIVES aims at locally identifying a voltage emergency situation, which is driven by the effect of the local LTC combined with all voltage controllers in the system. When the LTC exhausts its tap range voltage control is lost at the particular bus and it is no longer contributing to the developing voltage instability.

The recorded voltage magnitude samples are used to fit the linear regression model as described in section 3.1 It was found that to correctly identify the overall trend of the voltage trajectory, it is beneficial to continue sampling the voltage magnitude even when it temporarily enters the deadband. Hence, the fitting of the secondary voltage is stopped only if the voltage remains inside the deadband longer than a time $T_{\text {off }}$ indicating that the system long-term dynamics have reached an equilibrium (see $A L 9-19)$. 
When a sufficient number of samples is collected the linear regression model is fitted (see $A L 22$ ). The slope $m_{i}$ of the fitted line is utilized in the evaluation process (see $A L 23$ ). A negative slope indicates that the voltage magnitude is declining and may indicate a deterioration of the system conditions, while a positive slope suggests an overall recovery of the voltage and improvement of the system conditions. Consequently, if the determined slope is positive, the controller is reset and issued warnings/alarms are cleared (see $A L 23-25$ ). In the case that the determined slope is negative, it is compared to the negative slope $m_{i-1}$, which was determined with the previous sample. A warning (eLIVES Warning) is issued, if the current slope $m_{i}$ is more negative then the slope $m_{i-1}$ (see $A L 31-33$ ).

For improving the performance of the method, the evaluation of $m_{i}$ and $m_{i-1}$ is delayed by $T_{\max }$ (see $A L 30$ ). The delay was chosen to give the LTC time to restore the voltage and to allow revelation of the overall voltage trend.

\subsection{2. eLIVES Alarm}

After an eLIVES Warning has been raised, the voltage on the primary side $V_{H V}$ of the transformer is used to determine, if an eLIVES Alarm should be issued. Subsequently, this alarm may be used to trigger corrective actions such as undervoltage load shedding, reverse tapping, etc. An eLIVES Alarm is triggered, if an eLIVES Warning was issued and the voltage magnitude on the primary side dropped by more than $\Delta V_{\text {crit }}$ (e.g. $0.05 \mathrm{pu}$ ) with respect to its value at $V_{H V}\left(t_{r e c}\right)$ (see $\left.A L 38-40\right)$. This acts as a safeguard requiring the observation of a certain voltage drop on the primary side before corrective actions are executed and it serves as an intentional, but indirect, delay while the voltage drop is not yet significant enough to take corrective actions.

\subsubsection{HV Alarm}

This alarm is triggered, when the voltage magnitude of the primary side falls below a critical value defined by the system operator, e.g. $V_{H V \text { Alarm }}=0.9 \mathrm{pu}$. 
Contrary to eLIVES Alarm, it is also triggered when the LTC succeeds in restoring the voltage on the secondary side, but the successive tap changes led to unacceptable low voltage on the primary side (see $A L 42-44$ ).

\subsubsection{Parameters of eLIVES}

eLIVES requires only few parameters, namely $T_{o f f}, \Delta V_{\text {crit }}$ and $V_{H V A l a r m}$. If eLIVES was activated, but the controlled voltage has been restored and has re-entered the deadband, $T_{\text {off }}$ corresponds to the time that eLIVES remains active. This time constant should be chosen long, e.g. $250.0 \mathrm{~s}$, to account for delays of other voltage controllers in the system. $\Delta V_{\text {crit }}$ corresponds to the voltage drop on the HV side, which should be observed before emergency control actions are triggered. The HV alarm serves as a back-up alarm and, hence, the threshold $V_{H V A l a r m}$ can be chosen low, e.g. $0.9 \mathrm{pu}$. The $\Delta V_{\text {crit }}$ and $V_{H V A l a r m}$ parameters are used quite often by Transmission System operators in static security assessment of their grids. Although they vary from one system to another, the values used in this paper are quite representative.

Other needed parameters are obtained from the settings of the respective LTC, such as the maximum tap delay $T_{\max }$, the width of the half-deadband $\delta$ and the lower hard tap limit $N_{\min }$.

\section{Simulation results - Identification of emergency situations}

\subsection{IEEE Nordic Test System}

In order to demonstrate the dependability and security of the proposed

eLIVES method, the Nordic test system proposed by the IEEE Task Force on Test Systems for Voltage Stability and Security Assessment is utilized. The detailed data of the test system as well as the operating points can be found in [15]. A one-line diagram can be seen in Fig. 5 .

As described in [15] all MV loads are served through distribution transformers equipped with LTC. The width of the half-deadband $\delta$ is equal to $0.01 \mathrm{pu}$. Contrary to [15] the LTCs do not have constant tapping delays, but variable 


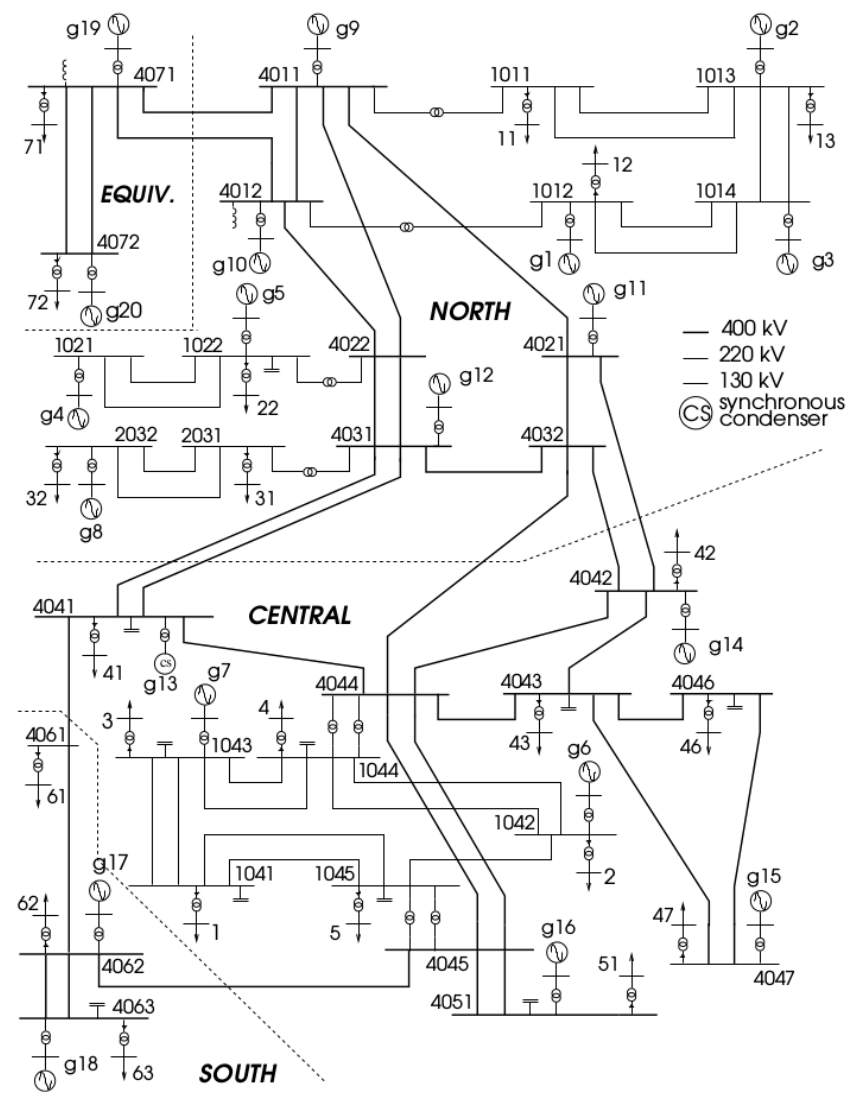

Figure 5: One line diagram of the IEEE Nordic test system [15]

delays with an inverse time characteristic. Hence, the tap delay is dependent on the observed voltage deviation; a larger deviation results in a shorter delay and vice versa. An important parameter for an LTC with inverse time characteristic is the maximum tap delay $T_{\max }$. In the simulations, it was chosen in the range of $25-40 \mathrm{~s}$.

For the eLIVES controllers only few parameters need to be selected: $T_{o f f}=$ $250 \mathrm{~s}, \Delta V_{\text {crit }}=0.05 \mathrm{pu}$ and $V_{H V \text { Alarm }}=0.9 \mathrm{pu}$. Moreover, it is assumed that the individual controller samples local voltage measurements every $0.5 \mathrm{~s}$. 


\subsection{Scenarios and operating points}

The proposed method is tested on three scenarios, which are described in detail in [15]. In all scenarios the same disturbance was considered, which is a solid three-phase fault occurring at $t=1.0 \mathrm{~s}$ close to bus 4032 and which is cleared after $100 \mathrm{~ms}$ through tripping of line $4032-4044$.

- Scenario 1 - Operating point A: The fault causes long-term voltage instability and a system collapse.

- Scenario 2 - Operating point B plus 350 MW: Operating Point (OP) B is obtained from OP A, but modified to be secure with respect to the aforementioned disturbance. The adjustments are detailed in [15]. In OP B plus $350 \mathrm{MW}$, the loading of the Central region has been uniformly increased by a total of $350 \mathrm{MW}$. As a result, the case is marginally stable, which means that the system conditions are severely deteriorated, but the voltage magnitudes at all buses eventually settle at acceptable levels.

- Scenario 3 - Operating point B plus 375 MW: The loading in the Central region was uniformly increased by $375 \mathrm{MW}$. The case is marginally unstable, and the voltage magnitudes at some buses settle at unacceptable low values.

\subsection{Scenario 1 - Operating point A}

Figure 6 shows a selection of $\mathrm{HV}$ bus voltages. It can be observed that a collapse in voltage occurs in the Central area, while the voltages in the Northern and Southern areas are little affected. After the contingency, oscillations can be seen, which damp out after approximately $20 \mathrm{~s}$ when the system reaches a shortterm equilibrium. Subsequently, LTCs are acting to restore the voltages in the system and OELs are activated to protect the field windings of the respective generators. This eventually leads to a system collapse after less than $140 \mathrm{~s}$.

Table 1 shows the assessment results of eLIVES. The table lists the buses at which a warning or alarm was raised. As described in Section 3.2, an eLIVES 


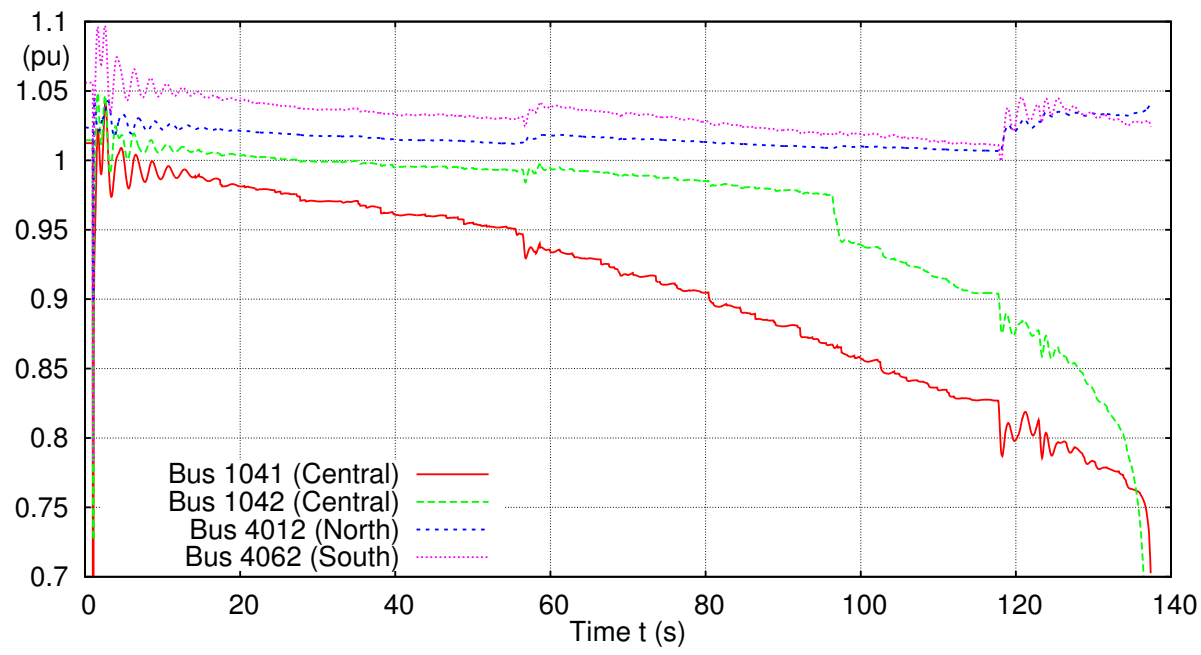

Figure 6: Scenario 1 - Selection of transmission voltages in Central, North and South region.

Warning is raised, when a negative trend of the controlled voltage $V_{c t l}$ is observed. Subsequently, an eLIVES Alarm is raised, if additionally the respective voltage on the primary side $V_{H V}$ dropped by more than $\Delta V_{c r i t}$. The HV Alarm serves as a backup, since it is solely triggered, if $V_{H V}$ drops below the pre-set value $V_{H V A l a r m}$.

The table shows that for a number of buses warnings were issued. These buses are either in the Central area $(1-5,41-43,46,47$ and 51) or are adjacent to the Central area (South: 62; North: $22 \& 31$ ). However, alarms have been issued almost exclusively at buses in the Central area. Moreover, it should be noted that the eLIVES Alarms were raised $15.8-38.5 \mathrm{~s}$ earlier than the HV Alarm at all buses. This shows a clear advantage of the eLIVES Alarm, since an earlier alert leaves more time to initiate counter actions.

Figure 7 displays the voltage evolutions at the MV bus 1 (red solid line) and the HV bus 1041 (green dashed line) as well as the ratio of transformer 1 - 1041 (blue dotted line) connecting these two buses. It can be observed that after a time delay the corresponding LTC attempts to restore the voltage by decreasing the transformer ratio. However, the LTC does not succeed in 
Table 1: Scenario $1 \&$ 3- Issued warnings and alarms.

\begin{tabular}{c|c|c|c||c|c|c} 
& \multicolumn{4}{|c||}{ Scenario 1 } & \multicolumn{3}{c}{ Scenario 3 } \\
\hline \multirow{2}{*}{ Bus } & eLIVES & eLIVES & $H V$ & eLIVES & eLIVES & $H V$ \\
& Warning & Alarm & Alarm & Warning & Alarm & Alarm \\
\hline \hline 1 & 58.2 & 58.2 & 80.8 & $-{ }^{2}$ & $-^{2}$ & 402.1 \\
\hline 2 & 89.0 & 97.8 & 118.2 & 719.5 & 755.8 & 835.5 \\
\hline 3 & 58.8 & 61.5 & 92.3 & $-{ }^{2}$ & $-{ }^{2}$ & 683.7 \\
\hline 4 & 66.5 & 67.0 & 84.6 & $-{ }^{2}$ & $-{ }^{2}$ & 716.2 \\
\hline 5 & 75.8 & 75.8 & 95.6 & 708.5 & 708.5 & 777.8 \\
\hline 22 & 98.9 & - & - & 400.6 & - & - \\
\hline 31 & 102.8 & 102.8 & - & - & - & - \\
\hline 41 & 80.81 & 80.8 & 118.2 & - & - & - \\
\hline 42 & 58.8 & 60.4 & 98.9 & $-{ }^{2}$ & $-{ }^{2}$ & 887.2 \\
\hline 43 & 67.6 & 74.2 & 112.1 & 796.5 & 796.5 & 854.2 \\
\hline 46 & 69.2 & 80.8 & 118.2 & 804.2 & 804.2 & 853.7 \\
\hline 47 & 86.3 & 102.8 & 118.7 & - & - & - \\
\hline 51 & 97.8 & 105.5 & 130.6 & - & - & - \\
\hline 62 & 92.3 & - & - & - & - & - \\
\hline
\end{tabular}

1 : Early warning at $56.6 \mathrm{~s}$

2 : Assessment with eLIVES was stopped since the LTC hit its limit. observed. Moreover, Fig. 7 shows that due to the inverse time characteristic of the LTC the tapping delay is decreasing with increasing depression of the voltage magnitude. eLIVES early identifies the negative trend of the voltage evolution and issues a warning at $t_{i}=58.2 \mathrm{~s}$. At this time, the voltage on the 360

HV side already fell by $0.05 \mathrm{pu}$ with respect to its value at $t_{r e c}$ and, hence, also the alarm is raised. Eventually, the HV voltage falls below $0.9 \mathrm{pu}$ and the HV Alarm is set at $t_{i}=80.8 \mathrm{~s}$.

Figure 8 shows the voltage evolution at the MV bus 31 (red solid line) and 


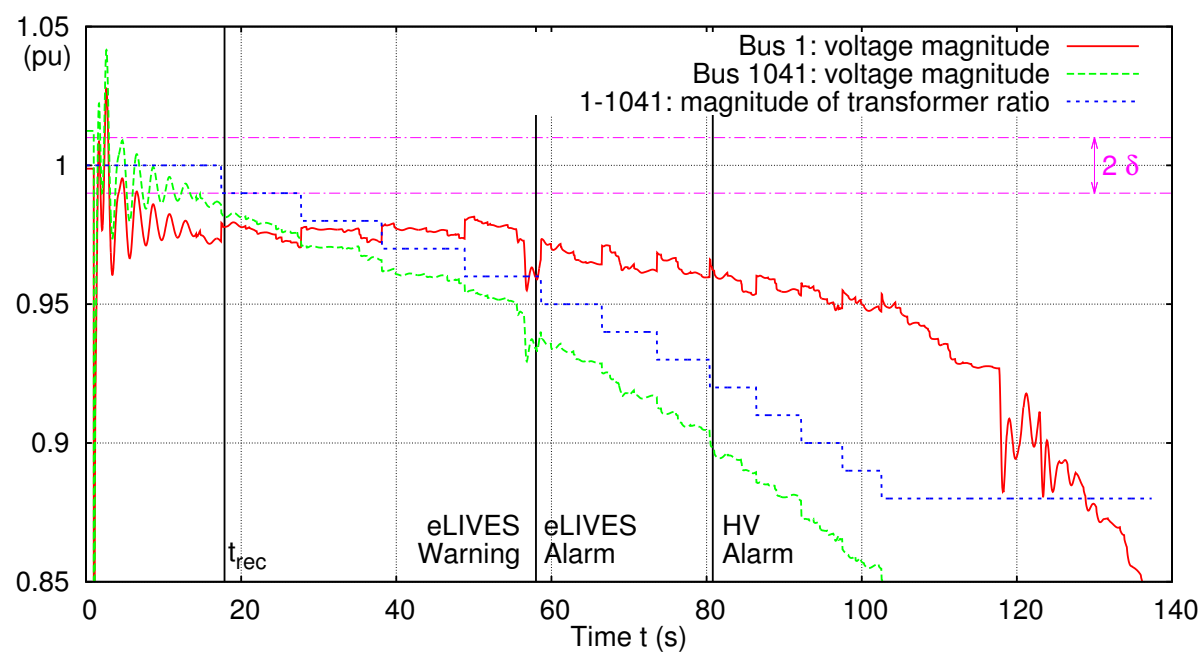

Figure 7: Scenario 1 - Bus voltage magnitude at HV bus 1041 and MV bus 1. Transformer ratio of transformer $1-1041$.

the HV bus 2031 (green dashed line), together with the transformer ratio (blue dotted line). Bus 31 is the only bus in Table 1 where an eLIVES Alarm is raised but no HV Alarm. The graph shows that the LTC does not succeed

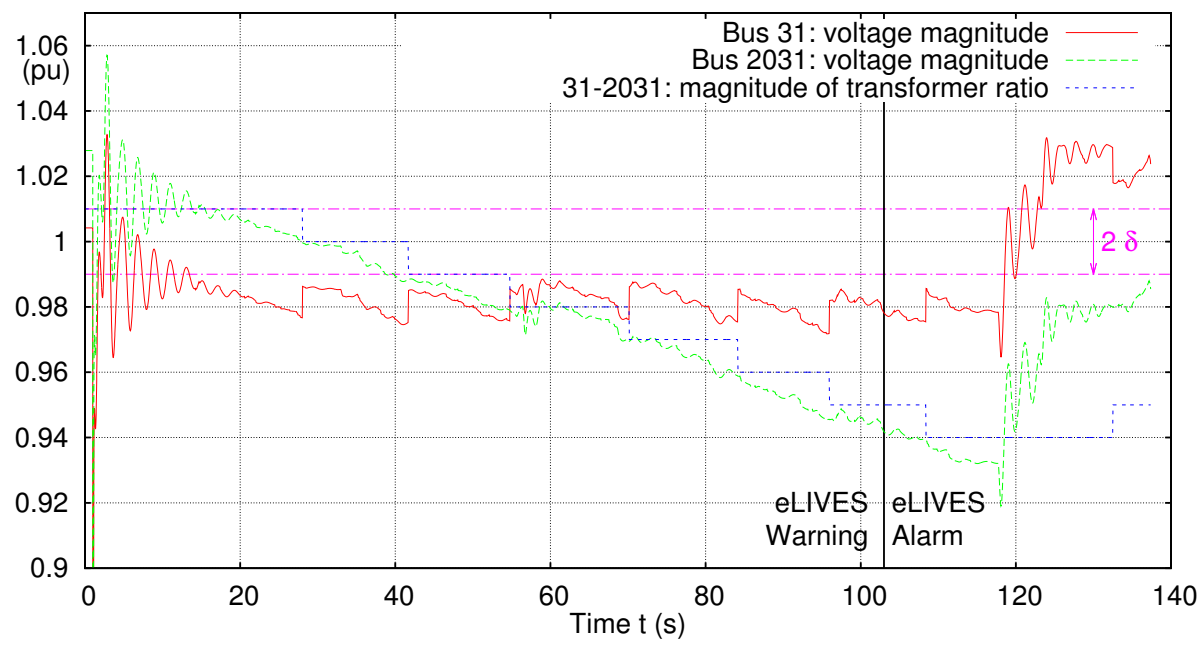

Figure 8: Scenario 1 - Bus voltage magnitude at HV bus 2031 and MV bus 31. Transformer ratio of transformer $31-2031$. 
in restoring the controlled voltage until $t_{i} \approx 118 \mathrm{~s}$. The voltage at the bus only recovers, when the voltage in the Central area collapses. Moreover, due to the repeated tap changes the HV voltage gets severely depressed. The eLIVES method detects a negative voltage trend and a serious depression of the HV voltage magnitude at $t=102.8 \mathrm{~s}$, which results in issuing of an eLIVES Warning and Alarm. Since the LTC $31-2031$ does not participate in the instability and the voltage at the controlled bus is eventually restored, this case is considered to be a false alarm. However, this false alarm is raised only after nine real alarms and any countermeasure taken in the mean time at any of these nine buses could avoid it.

\subsection{Scenario 2 - Operating point B plus $350 \mathrm{MW}$}

In this marginally stable case, the proposed method does not raise any warnings or alarms. This is due to the fact that all voltages in the system can be restored to an acceptable level. Figure 9 shows the successful restoration of the

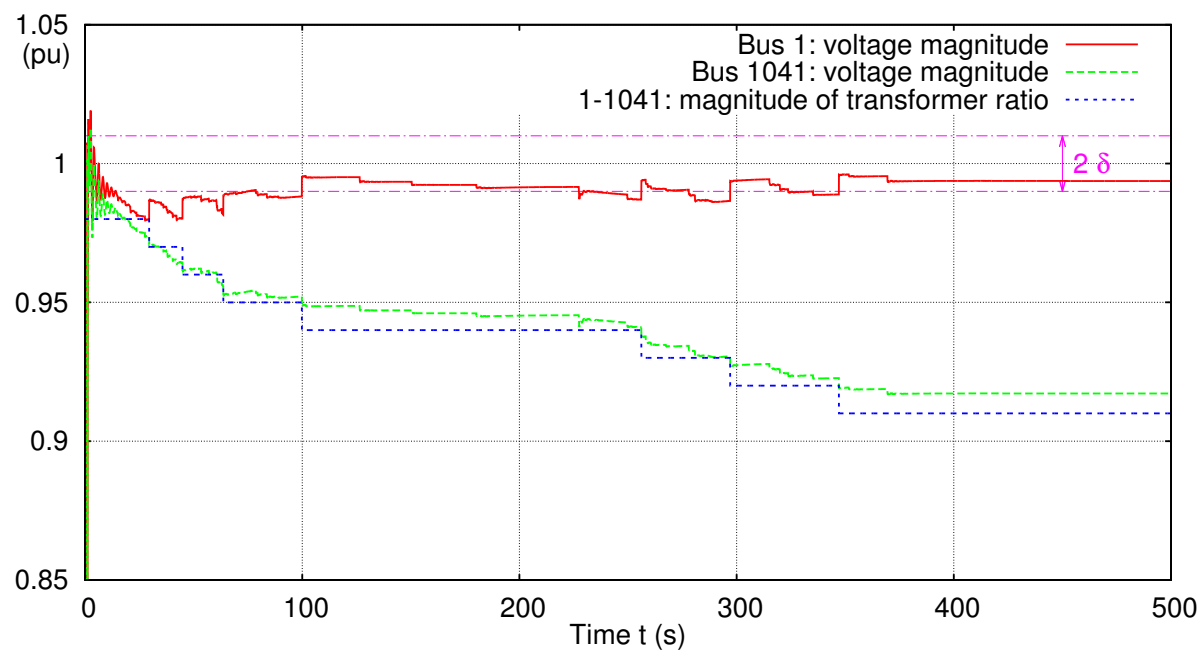

Figure 9: Scenario 2 - Bus voltage magnitude at HV bus 1041 and MV bus 41. Transformer ratio of transformer $1-1041$.

380

voltage at bus 1 . 


\subsection{Scenario 3-Operating point B plus $375 \mathrm{MW}$}

This scenario represents a marginally unstable case, which initially appears to be stable but instability is revealed in the Central area after $700 \mathrm{~s}$. Table 1

lowest tap position is reached. Then the voltage at the bus is not any longer controlled. As described in Section 3.2, eLIVES is subsequently deactivated after a time delay. Figure 10 shows the voltage magnitude at bus 1, bus 1041 and the ratio of transformer $1-1041$. The voltage at bus 1 initially improves due

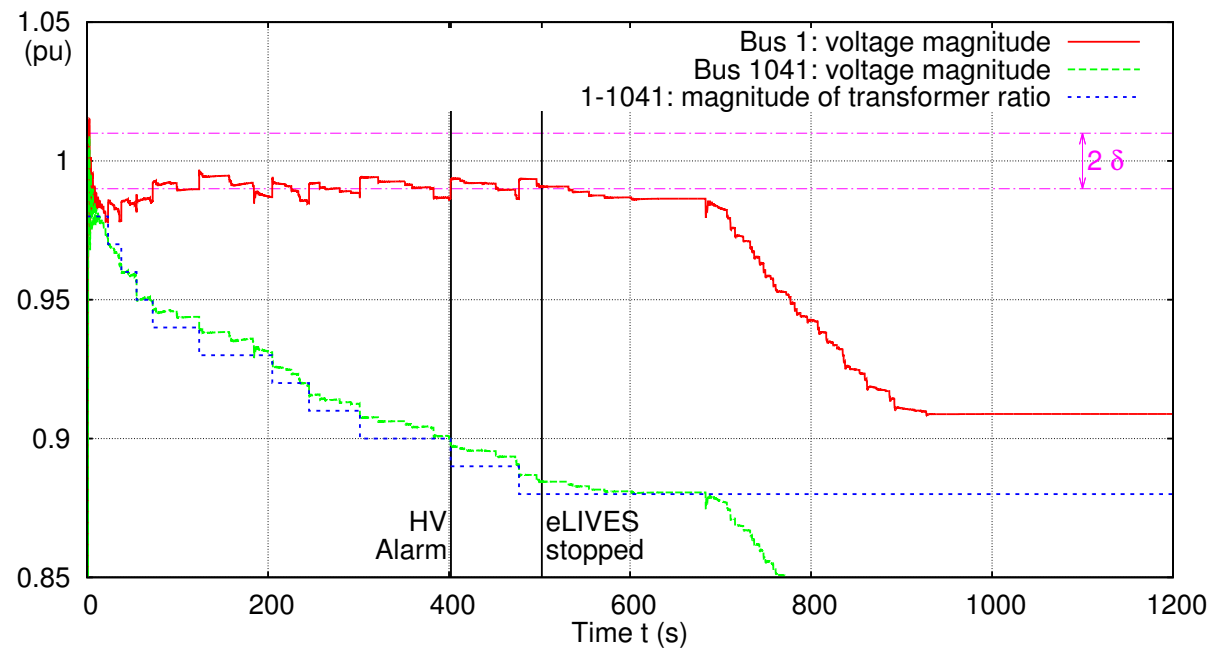

Figure 10: Scenario 3 - Voltage magnitude at HV bus 1041 and MV bus 1. Transformer ratio of transformer $1-1041$.

to the repeated tapping action, which results in a general positive voltage trend and, hence, no eLIVES Warning is raised. However, the voltage at bus 1041 is getting significantly depressed and drops below the critical value of $0.9 \mathrm{pu}$, which is considered unacceptable. This causes the HV Alarm to be raised. 
At $t=477.3$ a last tap change occurs and the transformer hits its lower tap limit. The monitoring with eLIVES is continued for the period of $T_{\max }=25 \mathrm{~s}$ and is stopped at $t=502.6 \mathrm{~s}$ as expected. The actual critical decrease of the voltage at bus 1 begins at around $680 \mathrm{~s}$, where the local LTC has already hit its limit. Consequently, voltage instability is driven by the other LTCs, causing the transmission voltages to drop.

Also for bus 42 only an HV Alarm was triggered, but no warning or alarm from eLIVES was raised. Figure 11 depicts the voltage at the MV bus 42 and at the HV bus 4042. Again the ratio of transformer $42-4042$ is displayed as well. The plot shows that the LTC repeatedly succeeds in bringing the

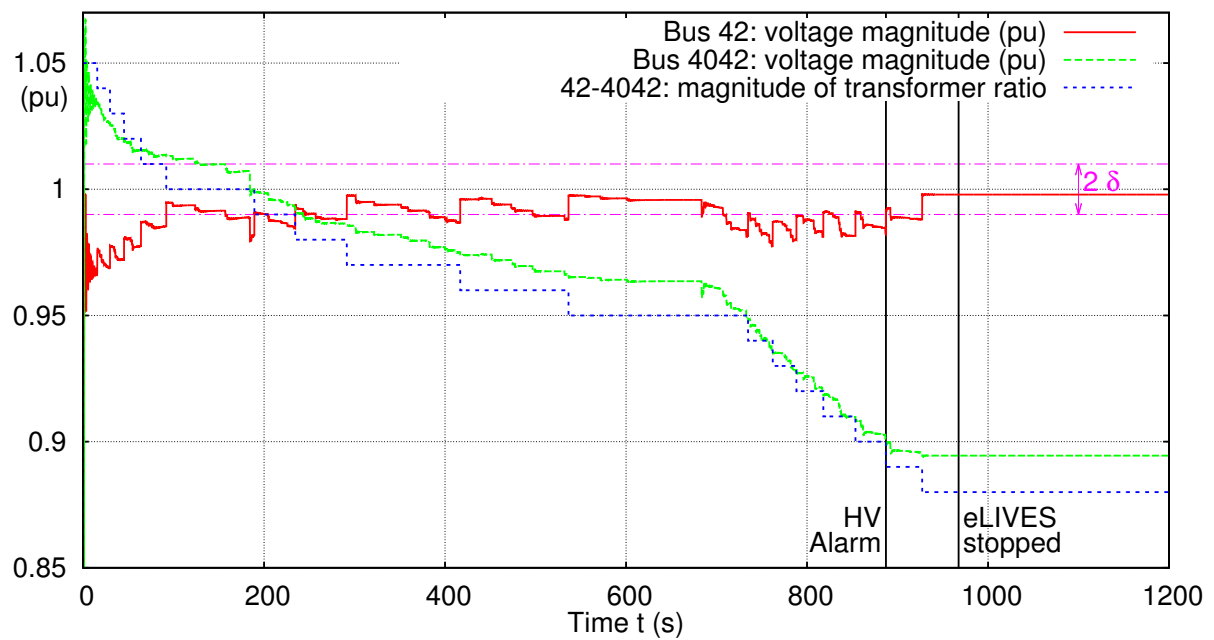

Figure 11: Scenario 3 - Voltage magnitude at HV bus 4042 and MV bus 42. Transformer ratio of transformer $42-4042$.

voltage magnitude at the controlled bus back into the deadband. Even after $700 \mathrm{~s}$, where at buses 1 a declining MV voltage magnitude can be observed, the voltage trajectory of bus 42 shows generally a slight increase leading eventually to a restoration of the voltage. Since the LTC performs satisfactory and due to the general positive trend, eLIVES does not raise a warning and, consequently, no alarm, as expected. However, the multitude of tap changes causes the HV voltage magnitude to decrease and fall below $0.9 \mathrm{pu}$, which causes the triggering 
of the HV Alarm.

Figure 12 displays the evolution of the voltage magnitudes at the MV bus 2 and HV bus 1042. Furthermore, the ratio of transformer $2-1042$ is shown. It can be observed that until approximately $700 \mathrm{~s}$ of simulation time, the LTC

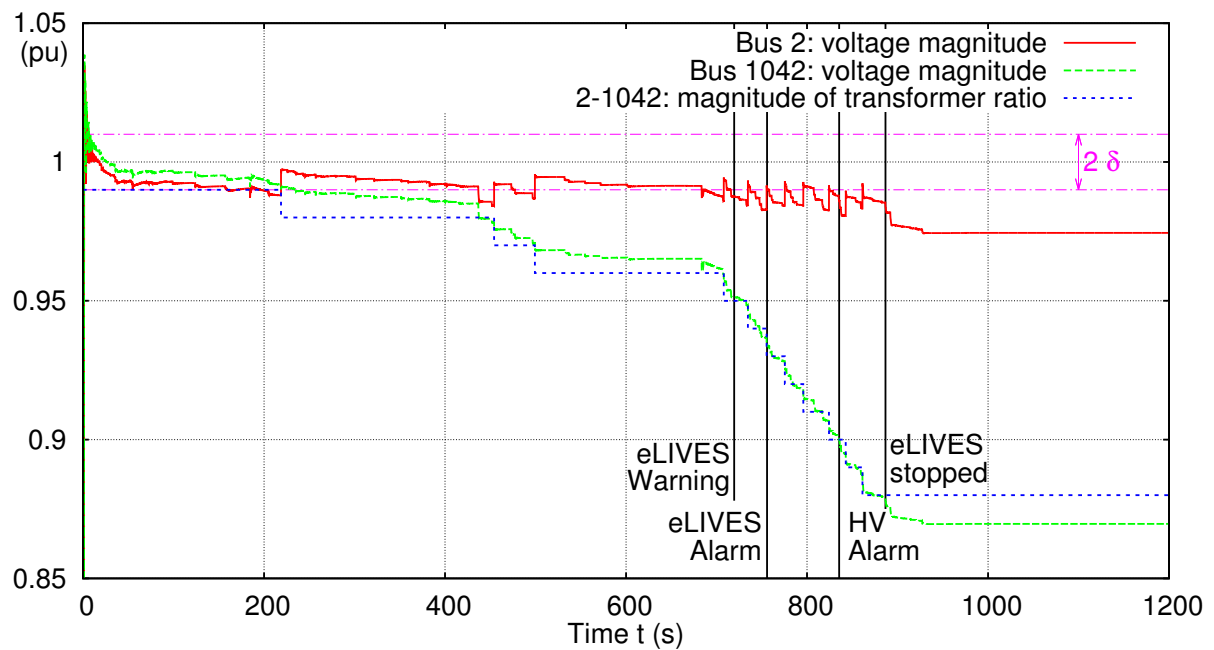

Figure 12: Scenario 3 - Voltage magnitude at HV bus 1042 and MV bus 2. Transformer ratio of transformer $2-1042$.

succeeds with only few tap changes to bring the controlled voltage back into the deadband. After $700 \mathrm{~s}$ and despite repeated tapping, the LTC can no longer restore the voltage at bus 2. At $719.5 \mathrm{~s}$, eLIVES identifies a negative trend of the voltage magnitude evolution and a warning is raised. The repeated actions of the LTC, which reduces the transformer ratio without boosting the voltage 425 magnitude on the secondary side, lead to a depression of the voltage at bus 1042, which eventually triggers the eLIVES Alarm at $755.8 \mathrm{~s}$ and the HV Alarm at 835.5 s. Consequently, eLIVES detects the detrimental condition $79.7 \mathrm{~s}$ earlier than the HV Alarm, which solely considers the HV voltage magnitude. Eventually, transformer $2-1042$ exhausts its tap range at $861.2 \mathrm{~s}$, which results in deactivating eLIVES at $886.7 \mathrm{~s}$. 


\section{Simulation results - Application to emergency control}

This section illustrates a potential application of the eLIVES Alarm. The emergency control scheme is of the type described in [16], but modified to replace the central alarm decision by the local eLIVES Alarm detailed in this paper. When an eLIVES Alarm is issued, the emergency control scheme is activated at the respective bus and blocks of load are shed in several steps. At activation, the voltage at the $\mathrm{HV}$ side is stored in $V_{s h}$ and used as shedding criteria. Afterwards, each time when $V_{H V}\left(t_{i}\right)$ falls below $V_{s h}$ a load block is shed. In this scenario, the block size was chosen to be $\Delta P=25 \mathrm{MW}$ (plus a corresponding decrease of the reactive power). After the eLIVES Alarm was raised, there is no additional delay for the initial load shedding. However, before each subsequent load shedding, a minimum time delay of $3 \mathrm{~s}$ is assumed.

The proposed method dynamically sets a voltage threshold used for the load shedding decision, while a standard under-voltage relay utilizes a pre-defined static voltage threshold as decision criterion. In practice, it may be challenging to determine such a static threshold, which is valid in a broad variety of system operation conditions. Indeed, to avoid tripping in non-critical situations, this threshold should be chosen low enough; on the other hand, setting it too low results in greater degradation of operation and may lead to shed more. As the proposed method allows identifying this threshold dynamically (at the time of the eLIVES Alarm), it can be at the heart of an adaptive system integrity protection scheme.

The effectiveness of eLIVES in combination with this load shedding scheme is presented on Scenario 1. The load curtailment succeeds in stabilizing the system. For that purpose, the scheme was activated at two buses, namely bus 1 and 42. In total $225 \mathrm{MW}$ load were curtailed to save the system (50 MW at bus 1 and $175 \mathrm{MW}$ at bus 42). Figure 13 shows the voltage evolutions at bus 1 and 1041 as well as the ratio of transformer 1 - 1041, when the emergency scheme is applied. As shown in Section 4.3 the eLIVES Alarm at bus 1 is triggered at $t=58.2 \mathrm{~s}$ (see also Table 1). The voltage threshold for load shedding $V_{s h}$ is set 


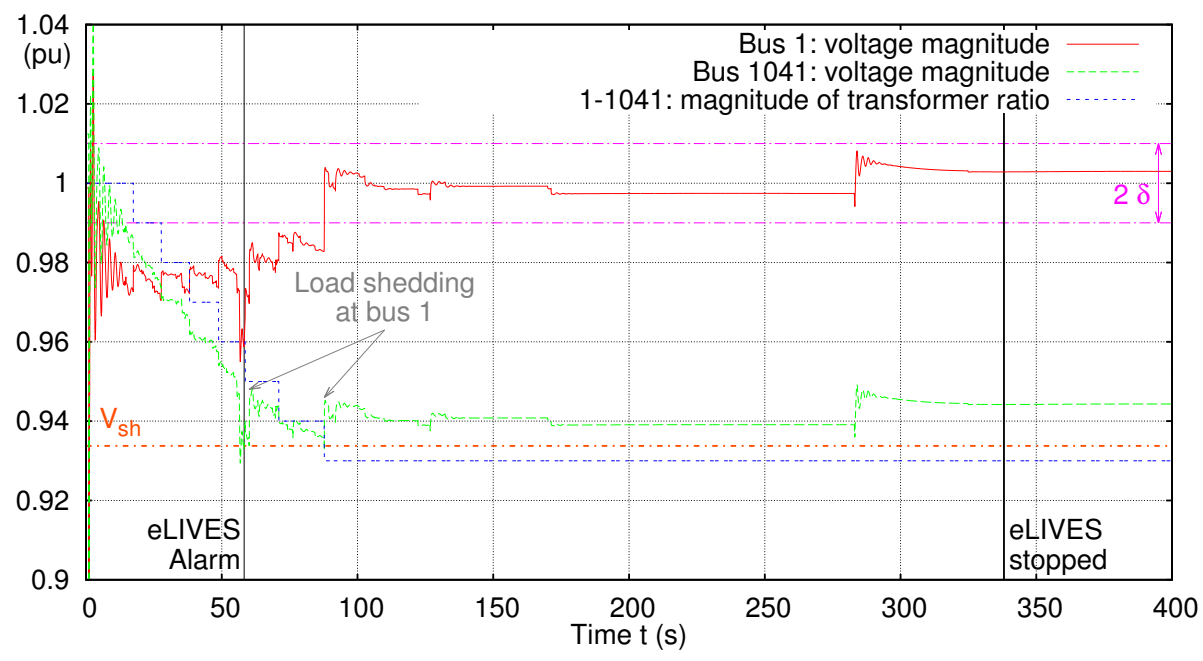

Figure 13: Scenario 1 with load shedding - Voltage magnitude at HV bus 1041 and MV bus 1. Transformer ratio of transformer $1-1041$.

equal to the value $V_{H V}(t=58.2)$, which is displayed as the orange dash-dotted line in Fig. 13. Shortly after at $t=60.1 \mathrm{~s}$, the first block of load is shed, which results in a step increase of the voltage magnitude at bus 1041. Subsequently, the voltage $V_{H V}$ drops one more time below $V_{s h}$ resulting in load shedding at time $t=87.8 \mathrm{~s}$ (see Fig. 13). Finally, $V_{H V}$ remains above $V_{s h}$ and a new steady state is reached. It should be mentioned that the voltage magnitude at bus 1 reenters the deadband after the second load shedding and settles at a value inside the deadband. Consequently, no reverse tapping of the LTC is observed. In this case, reverse tapping could indicate that the load shedding at the respective bus was excessive, which led to an overshoot of the MV voltage. Moreover, the monitoring with eLIVES is terminated at $t=338.6 \mathrm{~s}$, since the distribution voltage stayed inside the deadband for longer than $T_{\text {off }}=250 \mathrm{~s}$.

\section{Simulation results - Robustness to measurement noise}

In this section, the robustness of the proposed method with respect to noise 475 on the input signal is assessed. The noise added to the signal could influence the 
time when an eLIVES Warning, eLIVES Alarm or an HV Alarm is detected. Since the additional condition $\left(V_{H V}\left(t_{r e c}\right)-V_{H V}\left(t_{i}\right)>0.05\right)$ for the eLIVEs Alarm as well as the sole condition $\left(V_{H V}\left(t_{i}\right)<V_{H V \text { Alarm }}\right)$ for the HV Alarm are expected to not be sensitive to noise, it was decided to focus on the investigation of the robustness of the eLIVES Warning. For that purpose, 1000 Monte-Carlo simulations of Scenario 1 were carried out, where noise was added to the voltage samples at bus 1 . The noise signal was generated by drawing samples from a Gaussian distribution, where the mean was set to $\mu_{\text {noise }}=0.0 \mathrm{pu}$ and the standard deviation $\sigma_{\text {noise }}=0.005 \mathrm{pu}$. The resulting voltage evolutions were assessed with eLIVES and the cumulative probability of the time at which the eLIVES Warning was raised is shown in Fig. 14.

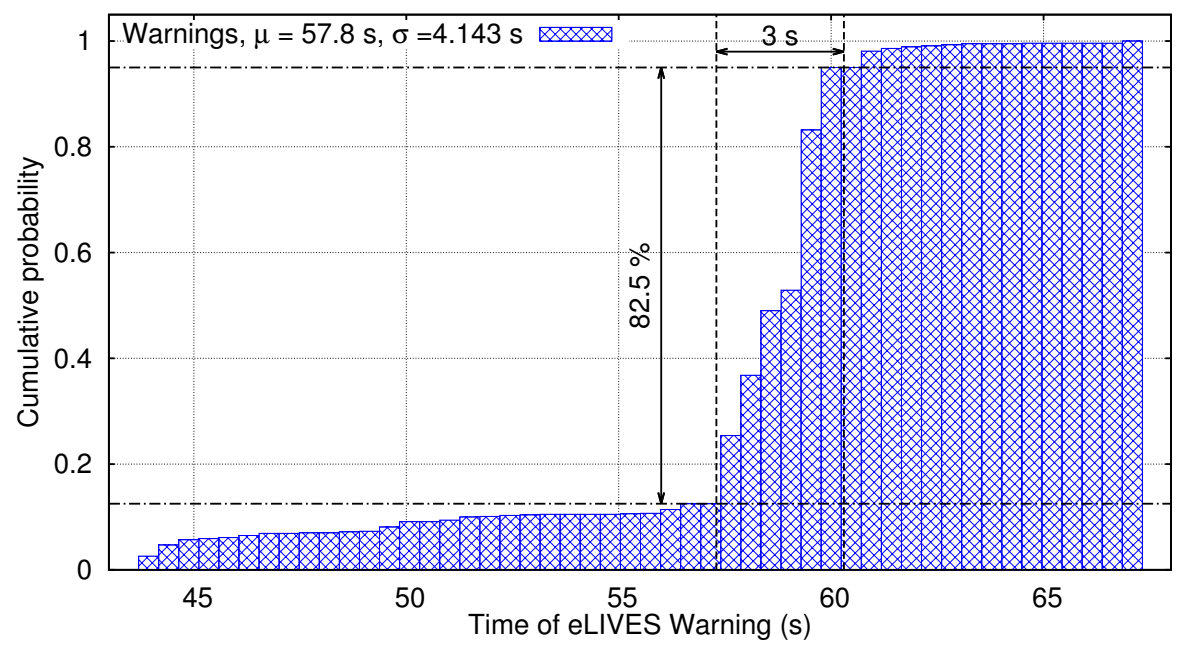

Figure 14: Scenario 1 - Cumulative probability of time of eLIVES Warning at bus 1 , when noise is added to the voltage measurements.

It should be noted that in all cases an eLIVES Warning was raised in spite of the added noise and, hence, no false negative errors occurred. The mean of the time at which the eLIVES Warning was issued is $57.8 \mathrm{~s}$, which is fairly close to the value obtained in the absence of noise (58.2 s see Table 1). Finally, it should be emphasized that $82.5 \%$ of the obtained warning times are in a narrow $3 \mathrm{~s}$ interval between $57.3 \mathrm{~s}$ and $60.3 \mathrm{~s}$. 


\section{Conclusion}

In this paper the eLIVES method was proposed, which allows a local, early

495 and dependable identification of long-term voltage instability.

In order to identify a developing instability, the method monitors the evolution of the LTC controlled voltage using a linear regression model. The model is fit to the whole set of measurements with a recursive least squares approach. This allows considering the voltage evolution over the entire time period after the deadband was left, but without the need to store a long sequence of values.

Finally, the slope is monitored and additional safeguard rules are checked to identify if a voltage emergency situation is entered.

The proposed method was tested on the IEEE Nordic Test System. The results from three scenarios were presented and discussed. They show that eLIVES can identify emergency situations reliably and early. It was shown that the eLIVES Warning accurately detects when the controlled voltage exposes a negative trend. Subsequently, when the voltage on the primary side of the LTC transformer has dropped by more than a pre-defined value, the eLIVES Alarm is issued, which correctly indicates a voltage emergency situation. Additionally, an HV Alarm acts as a back-up. The proposed method did neither raise any false alarms in the stable case, nor false alarms at non-critical buses in the unstable cases. Finally, a successful application to load shedding was reported.

Among the future investigations, it is planned:

- to assess the capability of eLIVES to control the successive corrective actions (as a substitute to monitoring a transmission voltage threshold);

- to embed the eLIVES method into an agent-based approach with limited communication between agents. This could result in an earlier identification of a voltage emergency situation, e.g. solely based on eLIVES Warnings.

- to investigate if a higher measurement sample rate and a filtering of the samples improves the performance of eLIVES in presence of noise. 


\section{References}

[1] T. Ohno, S. Imai, The 1987 tokyo blackout, in: 2006 IEEE PES Power Systems Conference and Exposition, 2006, pp. 314-318. doi:10.1109/ PSCE. 2006.296325,

[2] C. W. Taylor, Power system voltage stability, The EPRI Power System Engineering Series, McGraw-Hill Ryerson, Limited, 1994.

[3] T. Van Cutsem, C. Vournas, Voltage stability of electric power systems, Kluwer, 1998, Springer, 2008.

[4] M. Glavic, T. Van Cutsem, A short survey of methods for voltage instability detection, in: 2011 IEEE Power and Energy Society General Meeting, 2011, pp. 1-8. doi:10.1109/PES.2011.6039311.

[5] R. Diao, K. Sun, V. Vittal, R. J. O'Keefe, M. R. Richardson, N. Bhatt, D. Stradford, S. K. Sarawgi, Decision tree-based online voltage security assessment using pmu measurements, IEEE Transactions on Power Systems 24 (2) (2009) 832-839. doi:10.1109/TPWRS.2009.2016528.

[6] M. Glavic, T. Van Cutsem, Wide-Area Detection of Voltage Instability From Synchronized Phasor Measurements. Part I: Principle, IEEE Transactions on Power Systems 24 (3) (2009) 1408-1416. doi:10.1109/TPWRS. 2009.2023271

[7] C. Bai, M. Begovic, R. Nuqui, D. Sobajic, Y. Song, On voltage stability monitoring with voltage instability predictors, in: 2013 IREP Symposium Bulk Power System Dynamics and Control - IX Optimization, Security and Control of the Emerging Power Grid, IEEE, 2013, pp. 1-8. doi: 10.1109/IREP . 2013.6629356

[8] A. Perez, H. Jóhannsson, J. Østergaard, Evaluation of enhancements to thevenin equivalent based methods for real-time voltage stability assessment, in: Proceedings of 5th IEEE PES Innovative Smart Grid Technologies, IEEE, 2014, pp. 1-5. doi:10.1109/isgteurope.2014.7028805. 
[9] K. Vu, M. M. Begovic, D. Novosel, M. M. Saha, Use of local measurements to estimate voltage-stability margin, IEEE Transactions on Power Systems 14 (3) (1999) 1029-1035. doi:10.1109/59.780916.

[10] I. Šmon, G. Verbič, F. Gubina, Local voltage-stability index using tellegen's theorem, IEEE Transactions on Power Systems 21 (3) (2006) 1267-1275. doi:10.1109/TPWRS.2006.876702.

[11] G. N. Taranto, C. Oyarce, S. Corsi, Further investigations on a phasor measurement-based algorithm utilized for voltage instability awareness, in: 2013 IREP Symposium Bulk Power System Dynamics and Control - IX Optimization, Security and Control of the Emerging Power Grid, Ieee, 2013, pp. 1-8. doi:10.1109/IREP.2013.6629381.

[12] C. D. Vournas, T. Van Cutsem, Local identification of voltage emergency situations, IEEE Transactions on Power Systems 23 (3) (2008) 1239-1248. doi:10.1109/TPWRS.2008.926425.

[13] C. D. Vournas, C. Lambrou, M. Kanatas, Application of Local Autonomous Protection Against Voltage Instability to IEEE Test System, IEEE Transactions on Power Systems (2015) 1-9doi:10.1109/TPWRS.2015.2489764.

[14] M. I. Jordan, Notes on recursive least squares, Tech. rep., University of California, Berkeley (1998).

[15] T. Van Cutsem (Chair), PES-TR19: Test Systems for Voltage Stability Analysis and Security Assessment, Tech. rep., IEEE PES Power System Dynamic Performance Committee (2015).

[16] B. Otomega, M. Glavic, T. Van Cutsem, A two-level emergency control scheme against power system voltage instability, Control Engineering Practice 30 (2014) 93 - 104. doi:10.1016/j.conengprac.2013.10.007. 